\title{
El Uso de la logica Difusa para la Potabilizacion del Agua
}

\author{
Duque Mauricio $^{1}$ Giraldo Eugenio ${ }^{2}$ Martínez Alba Cristina ${ }^{2}$ \\ Gauthier Alain ${ }^{4} \quad$ Villa José Luis ${ }^{5}$
}

\begin{abstract}
Resumen
El proceso central en una planta de potablización de agua es el de coagulación el cual se maneja frecuentemente en forma manual. Este trabajo presenta una alternativa de control automático de estos procesos, la cual usa la teoría de conjuntos difusos. Se aplicó a la planta Wiesner de Bogotá que suministra cerca del $70 \%$ del agua de la ciudad, con muy buenos resultados con respecto a la turbiedad del agua filtrada que se entrega a la red de distribución. Se hizo uso de un equipo denominado SCD (sreaming current detector) el cual permite obtener una medida del estado de carga de las partículas en suspensión y de esa manera calcular la dosis del coagulante que se debe aplicar.

\section{Palabras clave:}

Coagulación, control automático, SCD, turbiedad, lógica difusa, filtración directa

\section{INTRODUCCIÓN}

El corazón de un sistema de potabilización de agua para consumo humano es el proceso de coagulación. En él se acondiciona el agua de tal forma que los procesos posteriores de limpieza, en donde, propiamente dicho, se remueven las impurezas, puedan realizar su función adecuadamente. Si la coagualación no funciona adecuadamente, tampoco funcionan adecuadamente los procesos posteriores de limpieza. Así de sencillo, si la coagulación no se optimiza tampoco se puede tratar el agua óptimamente. Pardójicamente, un proceso de tal importancia está lejos de ser operado de una manera óptima en las plantas de tratamiento de agua potable actualmente. Tradicionalmente la coagulación de las aguas que entran a las plantas de potabilización se realiza de una manera manual, y con controles fuera de linea que no permiten ajustar adecuadamente las dosis de las sustancias coagulantes a los cambios que tiene la planta tanto en cantidad como en calidad del agua que llega.

La dosificación automática de coagulantes no es de niguna manera un proceso fácilmente automatizable debi-

1 Profesor Asociado, Departamento de Ingeniería Eléctrica y Electrónica, Universidad de Los Andes.

2 Profesor Asociado, Departamento de Ingeniería Civil y Ambiental, Universidad de Los Andes.

3 Estudiante de Magíster, Departamento de Ingeniería Eléctrica y Electrónica, Universidad de Los Andes.

4 Profesor Titular, Departamento de Ingeniería Eléctrica y Electrónica, Universidad de Los Andes..

5 Estudiante de Doctorado 
do en primera instancia a la dificultad de poder definir, en linea, bien sea por mediciones directas o por estimaciones indirectas, cuando se logró una adecuada coagulación del agua cruda que llega a la planta. En muchos casos las variables de calidad del agua cruda que definen la dosis del coagulante no son fácilmente medibles en linea con sensores robustos y confinables como por ejemplo el color. Por otra parte la desestabilización de las sustancias y partículas que se quiere remover es difícil de obtener en linea, al igual que otras variables que definen la eficiencia del proceso de coagulación.

La planta Wiesner, es la principal planta de potabilización de la ciudad de Bogotá. Suministra cerca del 70\% del agua que consume la ciudad, y tiene un agua cruda de excelente características de calidad, lo que permite la aplicación del proceso de filtración directa, es decir, que luego de la coagulación se pasa directamente a los filtro saltándose los procesos clásicos de floculación y sedimentación. Desde el punto de vista de la coagulación, sinembargo, el agua es complicada para la definción de la dosis óptima del coagulante, precisamente por las características de buena calidad que la caracterizan como son la baja turbiedad, baja alcalinidad y alto color. En la actualidad la dosificación del coagulante, alumbre, se realiza de una manera manual, y la definción de la dosis se hace con base en los ensayos de jarras que son los procedimientos tradicionales para estas situaciones. En el presente trabajo se muestra los ensayos realizados como una primera aproximación a la automatización de la dosificación de coagulantes en la planta Wiesner, utilizando la lógica difusa para la generación del esquema de control.

Desde hace varios años han venido trabajando en dicha planta estudiantes del programa de magíster de ingeniería eléctrica en la automatización de este proceso de coagulación floculación. Como resultado de las tesis de grado, en el primer semestre del 2000 se desarrolló y probó un regulador basado en lógica difusa para el control automático de dicho proceso.

Se ha retenido la utilización de lógica difusa, pues el modelamiento del fenómeno usando ecuaciones diferenciales es bastante complejo y poco conocido, mientras los operarios tienen conocimientos empíricos para la operación manual. Estas dos características hacen de la lógica difusa un excelente candidato para el desarrollo de un posible regulador industrial.

Actualmente la dosificación de sulfato de aluminio (alumbre) se realiza mediante una rueda de canjilones la cual se compone de un motor de velocidad variable, que hace girar una rueda provista de una serie de canji- lones, que al pasar por su parte inferior se van llenando con la solución, la cual depositan en un elemento central por donde escurre el flujo hasta el punto de aplicación (Figura 1).

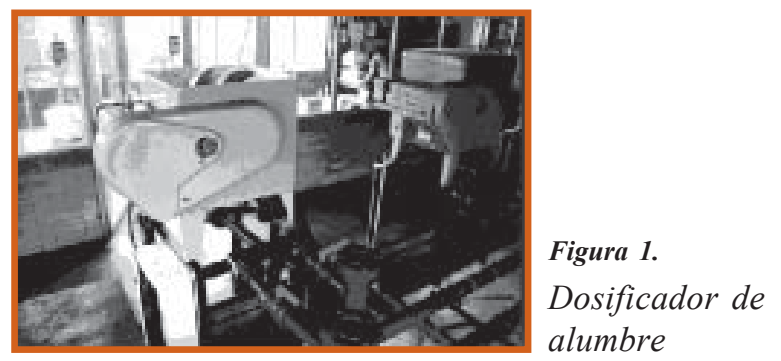

Desde el punto de vista clásico de control, se trata de un proceso con una dinámica lenta (en el rango de los minutos), con perturbaciones y cambios de puntos de operación lentos, aspecto que ha permitido un manejo manual de la dosificación en la mayoría de plantas, sin una clara señal objetivo, pues puede ser utilizada una mezcla entre turbiedad, SCD y carrera de los filtros.

\section{2. "Streaming Current Detector (SCD)"}

Una medida de que tan bien se han coagulado las partículas se puede lograr utilizando el denominado potencial Z. El potencial Z cuantifica que tan cargadas se encuentran las partículas en suspensión en el agua, es decir, las partículas que se pretende remover para limpiarla, bajo el supuesto que para remover éstas del agua es necesario que la carga sea nula o prácticamente nula. La función principal del coagulante es lograr que las cargas en las partículas, junto con la nube de iones que la rodean, sean prácticamente nulas. Tradicionalmente la medición del potencial $\mathrm{Z}$ se realiza tomando muestras del agua coagulada y analizándolas en el laboratorio, lo que puede tomar desde varios minutos a algunas horas. Existen sinembargo algunos aparatos que se han desarrollado para estimar en linea el potencial $\mathrm{Z}$ como el denominado SCD del ingles Streaming Current Detector.

El equipo SCD adquirido para el proyecto, toma una muestra continua de agua una vez dosificado el coagulante (alumbre), la cual es introducida en el analizador. Un pistón dotado de movimiento oscilatorio impulsa el paso del agua por la pared interior de la celda. Las partículas suspendidas se adhieren a las paredes debido a la acción de las fuerzas electrostáticas. Como la muestra se mueve rápidamente hacia adelante y hacia atrás, los iones que rodean las partículas cargadas, es 
decir los coloides, son llevados cerca de la superficie de la partícula y movidos a través de los electrodos. Así es inducido un diferencial de potencial entre los dos electrodos en la parte superior e inferior de la celda [4]. El potencial resultante, proporcional a la carga, es procesado electrónicamente para dar una lectura de la corriente de fluido en micro-amperios (streaming current units SCU). La figura siguiente muestra el equipo utilizado:

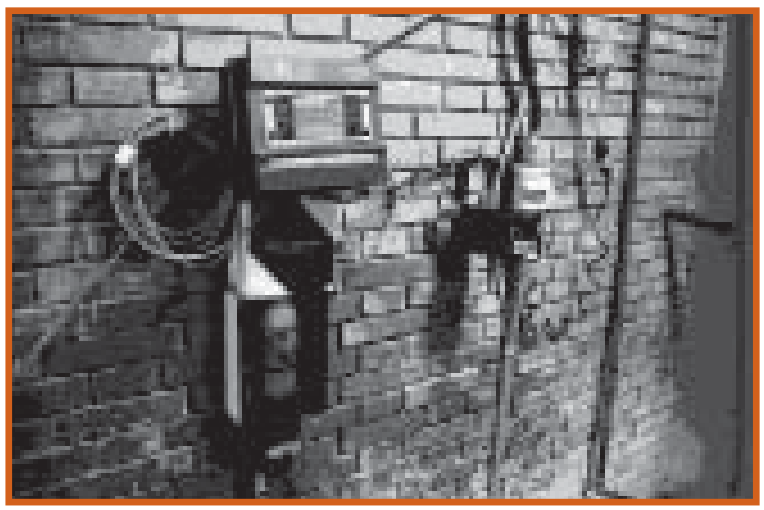

Figura 2: Equipo SCD

Este equipo es calibrado de tal forma que dé una lectura negativa si las partículas en suspensión están cargadas negativamente, y similarmente una lectura positiva para un sistema cargado positivamente. Entre mayor sea la magnitud de la corriente, mayor es la carga del sistema que está siendo medido y por consecuencia mayor es la repulsión entre las partículas en suspensión. De esta forma se puede conocer rápidamente el efecto de la dosificación suministrada.

\section{Sistema de Adquisición}

Para determinar la relación entre los factores que afectan el proceso (turbiedad de agua cruda y de agua filtrada, SCD, caudal, ...) se implementó una aplicación sobre LabView, la cual toma las señales en 4-20 mA de los transmisores de los sensores respectivos. Estos datos son guardados en un archivo tipo texto para su posterior análisis y diseño del sistema de control. El sistema de adquisición se muestra en la figura 3 .

\section{Control Por lógica Difusa}

El control difuso es una estrategia basada en una base de conocimientos, y un conjunto de reglas "difusas". Esta estrategia de control ha mostrado buenos resultados en contextos donde la obtención de un modelo suficientemente simple para el control resulta costosa o

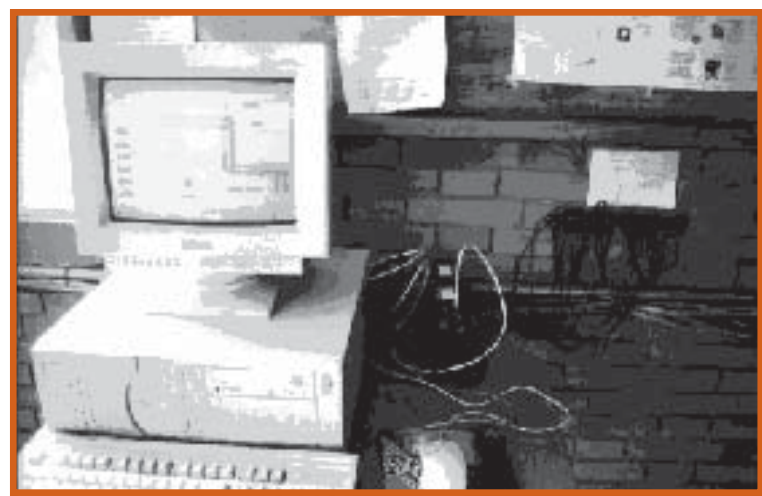

Figura 3: Sistema de adquisición utilizado

poco eficiente, pero en contrapartida se tiene el conocimiento para la operación manual.

De esta forma, se privilegia la utilización de conocimiento adquirido empíricamente sobre la forma en que trabaja el proceso y como se opera manualmente. Este conocimiento usualmente tiene una forma lingüística basada en apreciaciones de un operario "experto". Se realizaron los siguientes diseños con esta estrategia de control, los cuales se probaron sobre el sistema real.

El sistema finalmente desarrollado se puede apreciar en la siguiente figura:

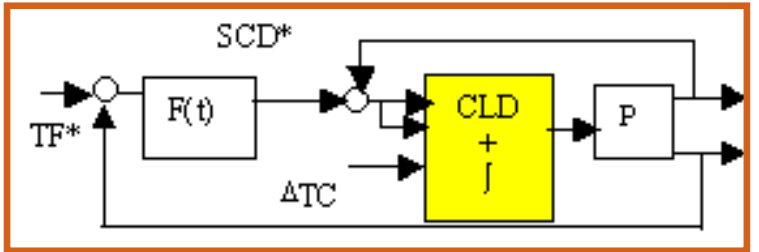

Figura 4: Esquema de control trabajado

Para este diseño, se tomó como valor de referencia la turbiedad de agua filtrada ( $\left.\mathrm{TF}^{*}\right)$, la cual se compara con la turbiedad de agua real cada 4 minutos, tiempo que según los datos tomados se alcanza a apreciar algún cambio después de cambiar la dosificación. La salida comparación entre el valor deseado y el real de turbiedad de agua filtrada (eTF), se relaciona con el valor deseado de SCD mediante una función que en el caso presentado es una línea recta cuya pendiente y corte se pueden modificar en línea según el punto de operación requerido.

Una vez hallado el valor de SCD* según el eTF, se realimenta el SCD real obteniendo el error de SCD (eSCD) y su variación (DESCD), estas dos últimas variables junto con la variación de turbiedad de agua cruda (DTC), son ahora las entradas al control difuso, cuyo tiempo de muestreo es de 60 segundos. 
La siguiente Figura ilustra la superficie para DTC cero.

Es decir que podemos tener un caso como:

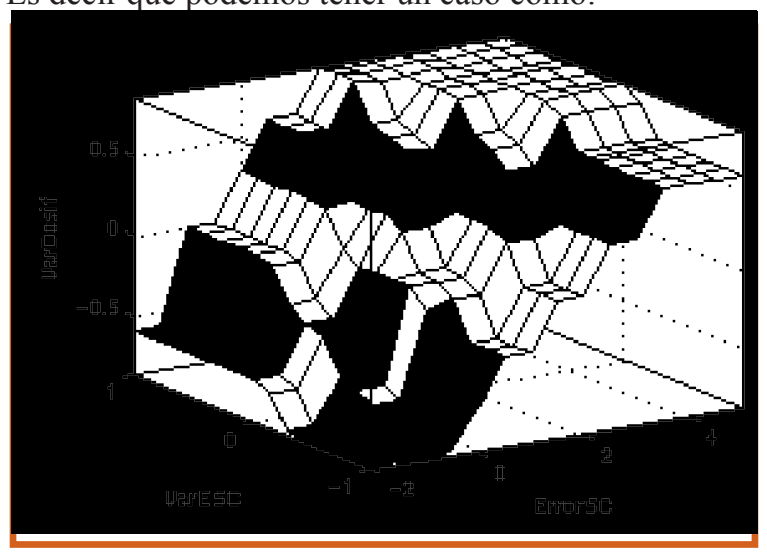

Figura 5 superficie de control para DTC cero.

Si eSCD es positivo grande (PG) y DESCD es negativo pequeño (PP) y DTC es cero $(Z)$, es decir que el valor real de SCD es más negativo que el deseado, pero la variación de este error está disminuyendo y la turbiedad de agua cruda no está cambiando; entonces la orden es: aumente un poco la dosificación (DDosif es positivo pequeño).

Tomando todas las posibles combinaciones, se diseñaron 175 reglas. Las Figuras 6 y 7 ilustran la respuesta del proceso controlado durante 6 horas continuas en modo automático.

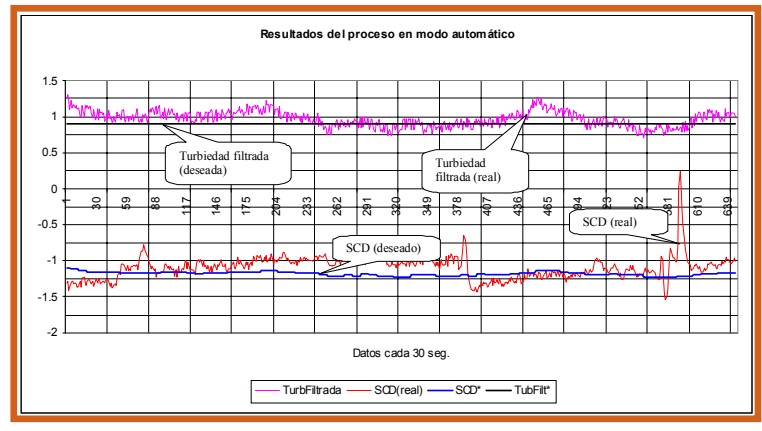

Figura 6. Respuesta de las variables del proceso en el modo automático

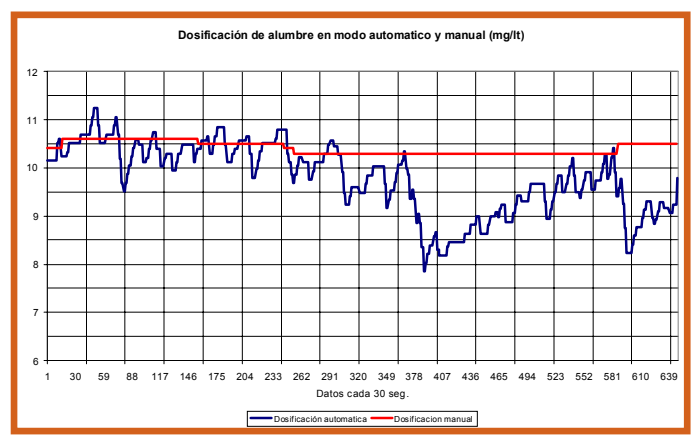

Figura 7. Comportamiento de la señal de control en el modo automático
En estas Figuras se observan los valores de: SCD, SCD deseado, turbiedad de agua real y deseada y el momento en el cual se inicia en modo automático. Las dos variables siguen la referencia en forma aceptable, se obtuvo un promedio de error de SCD de -0.0609 y varianza de 0.043 , lo cual es adecuado para el proceso. En el caso de la turbiedad de agua filtrada se obtuvo un error promedio de -0.088 y varianza de 0.016 el cual es muy conveniente, ya que esta es la variable de calidad que efectivamente se desea controlar.

En la muestra 581 se observa un cambio en el valor del SCD, debido a un aumento en el caudal de entrada y a un cambio en la calidad del agu ya que en ese momento se interrumpió la entrada de agua de la represa de San Rafael. A a los 8 minutos el control logra establecer nuevamente el valor de SCD que se requiere ya que éste realizó una disminución en la dosificación en ese momento.

En cuanto a la señal de control (Figura 7), la dosificación de alumbre en mg/lt, que el control decidió, se observan variaciones suaves buscando seguir las dos referencias, con un promedio de dosificación de $9.7 \mathrm{mg} / \mathrm{lt}$. Es importante anotar, que en el caso del manejo del proceso por un operador, éste no variaría la dosificación de una forma tan fina ni en intervalos de tiempo tan seguidos. La decisión del operador consistiría en mantener la dosificación, en este caso un promedio de $10.42 \mathrm{mg} / \mathrm{lt}$, esperando ver resultados después de 1 o 2 horas o cuando las condiciones de entrada cambien. En tal caso haría cambios más bruscos $( \pm 0.4 \mathrm{mg} / \mathrm{lt})$,

\section{Conclusiones y Recomendaciones}

El control por lógica difusa facilita para este tipo de procesos el diseño de un regulador apropiado. Se logró controlar adecuadamente la turbiedad del agua filtrada que se envía para consumo de los bogotanos optimizando la dosificación de los coagulantes, en este caso del alumbre.

Fue importante en la definición de las reglas de control la participación de los expertos de la planta y el conocimiento de las normas de calidad del agua y del comportamiento químico del alumbre como agente coagulante.

Manejar más de tres variables a la entrada de un control difuso cada una con varios conjuntos, puede volverse muy complejo ya que el número de reglas aumenta aumentando también la posibilidad de error al definirlas. Esto puede ser de importancia en un futuro cuando se complemente el presente sistema con la medición y el 
control del pH del agua coagulada.

La presente contribución es un trabajo preliminar, el cual se concentró en el control usando como variable objetivo el valor medido por el SCD en el lazo rápido, $\mathrm{y}$ la turbiedad en el lazo cerrado. El sistema se acopló vía una función lineal, la cual mostró funcionar como primera aproximación. Sin embargo, se requieren estudios posteriores, pues esta relación lineal, obtenida empíricamente, debe ser validada o replanteada desde el marco teórico.

\section{Agradecimientos}

Se agradece de manera especial la colaboración recibida por parte de la EAAB para la realización del presente trabajo y en especial de todos los trabajadores y directivas de la planta Wiesner por su apoyo incondicional y su confianza.

\section{BiBLIOgRAFÍA}

1 Valencia Arboleda, Jorge. Teoría y práctica de la purificación del agua, pág. 21-51. Acodal 1992
2 Conley, W.R. Coagulation Control, JAWWA, Vol60, 165,1968

3 Use of Aluminum Salts in Drinking Water Treatment. AWWA Government Affairs. April 11, 1997

4 Manual Micro 200 Streaming Current Detector, HF scientific, Inc.

5 Controlling Clarification using a Streaming Current Detector (SCD). 43rd Annual Meeting, International Water Conference, Pittsburg, Pennsylvania. October 25-27, 1982

6 Steven Dentel and Kristine M. Kingery. An Evaluation of Streaming Current Detectors. AWWA Research Foundation, November 1988. http:// www.awwarf.com/exsums/90536.htm

7 J.C. Liu and M.D. Wu. Fuzzy control of coagulation reaction through streaming current monitoring. Department of Chemical Engineering, National Taiwan Institute of Technology. 1997 